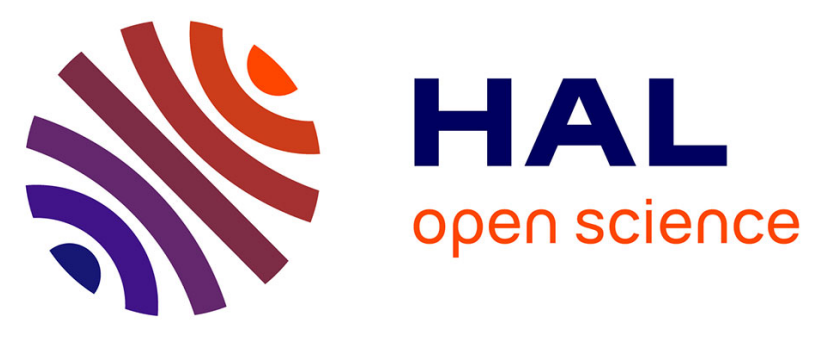

\title{
Evidence of the Superparamagnetic State in the Zero-Field Microwave Susceptibility Spectra of Ferrimagnetic Nanoparticles
}

Marie Darcheville, Anne-Lise Adenot-Engelvin, Christophe Boscher, Nicolas Vukadinovic, Christophe Lefèvre, André Thiaville, Clément Sanchez

\section{To cite this version:}

Marie Darcheville, Anne-Lise Adenot-Engelvin, Christophe Boscher, Nicolas Vukadinovic, Christophe Lefèvre, et al.. Evidence of the Superparamagnetic State in the Zero-Field Microwave Susceptibility Spectra of Ferrimagnetic Nanoparticles. IEEE Magnetics Letters, 2020, 11, pp.1-5. 10.1109/LMAG.2020.3026228 . hal-03008885

\section{HAL Id: hal-03008885 \\ https://hal.science/hal-03008885}

Submitted on 8 Dec 2020

HAL is a multi-disciplinary open access archive for the deposit and dissemination of scientific research documents, whether they are published or not. The documents may come from teaching and research institutions in France or abroad, or from public or private research centers.
L'archive ouverte pluridisciplinaire HAL, est destinée au dépôt et à la diffusion de documents scientifiques de niveau recherche, publiés ou non, émanant des établissements d'enseignement et de recherche français ou étrangers, des laboratoires publics ou privés. 


\title{
111Chapitre d'équation 1 Section 1Nanomagnetics
}

\section{Evidence of the superparamagnetic state in the zero-field microwave susceptibility spectra of ferrimagnetic nanoparticles}

\author{
Marie Darcheville ${ }^{1}$, Anne-Lise Adenot-Engelvin ${ }^{1}$, Christophe Boscher ${ }^{1}$, Nicolas Vukadinovic ${ }^{2}$, Christophe \\ Lefevre $^{3}$, André Thiaville ${ }^{4}$ and Clément Sanchez ${ }^{5}$ \\ ${ }^{1}$ CEA, DAM Le Ripault, F-37260 Monts, France \\ ${ }^{2}$ Dassault Aviation, Saint-Cloud, France \\ ${ }^{3}$ IPCMS, 67200 Strasbourg, France \\ ${ }^{4}$ Laboratoire de Physique des Solides, Univ. Paris-Saclay, CNRS UMR 8502, 91400 Orsay, France \\ ${ }^{5}$ Laboratoire Chimie de la Matière Condensée de Paris, UPMC, 75252 Paris Cedex 05, France
}

Received 1 Apr 2020, revised 15 Apr 2020, accepted 20 Apr 2020, published 1 Jun 2020, current version 15 Jun 2020.

\begin{abstract}
Static and dynamic magnetic properties of $\mathrm{Zn}_{0.4} \mathrm{Fe}_{2.6} \mathrm{O}_{4}$ nanoparticles synthesized by thermal decomposition have been investigated. Two ranges of diameter have been used: small particles (diameter about $6.2 \mathrm{~nm}$ ) and larger ones (diameter about 22.4 $\mathrm{nm}$ ). The nanoparticle microstructure has been characterized by transmission electron microscopy. The temperature dependence of the zero-field dynamic permeability for both nanoparticle sizes has been studied, revealing a superparamagnetic state for the small ones. Effects of the nanoparticle size on the dynamic permeability have been studied, and linked to the superparamagnetic state. A dynamic susceptibility model has been found to reproduce the experimental behavior as well as its temperature dependence.
\end{abstract}

Index Terms—Nanomagnetics, dynamic magnetic susceptibility, ferromagnetic resonance, nanoparticles, superparamagnetism.

\section{INTRODUCTION}

Magnetic iron oxide nanoparticles are widely studied for biomedical applications like markers for magnetic resonance imaging, new nanosystems for hyperthermia, or drug delivery carriers due to their low toxicity, and biocompatibility [Xiao 2020]. Their tunable sizes make them perfect candidates to observe or interact locally with entities at the cell scale without damaging healthy tissues [Nikiforov 2009]. They are designed with a high saturation magnetization to provide a sufficient heating of the particles under the applied AC fields [Bañobre-López 2013] and their size is small enough to ensure a superparamagnetic behavior: for small particle sizes, the thermal energy is close to the difference of magnetic energy between two magnetization directions resulting in fluctuations in the magnetization direction.

Such particles are also suitable for designing new magnetic materials for electromagnetic applications as a constituent of an ink, which will be then ejected by inkjet printing for elaborating wellcontrolled patterned coatings [Saleh 2017]. The small grain size inhibits complex magnetic textures and thus prevents losses arising from the domain wall resonance. Recently, a one-step synthesis and deposition process of nanoparticle derived from microwave-assisted thermal decomposition synthesis has shown successful compatibility with CMOS inductors enhancing the $\mathrm{Q}$ factor 20 times and extending the frequency range on the $\mathrm{C}$ band in the case of $\mathrm{Mn}_{0.5} \mathrm{Zn}_{0.5} \mathrm{Fe}_{2} \mathrm{O}_{4}$ composition [Sai 2019] or with partially inverted $\mathrm{ZnFe}_{2} \mathrm{O}_{4}$ ferrites [Sai 2017]. Following Song [2013], the size of the nanoparticle is believed to play a key role on the high frequency susceptibility through the superparamagnetic/ferromagnetic resonance [Brown 1963].

orresponding author: A-L. Adenot-Engelvin (anne-lise.adenot-engelvin@cea.fr). Digital Object Identifier: 10.1109/LMAG.XXXX.XXXXXXX (inserted by IEEE).
Shedding light on the size effect on the microwave susceptibility should allow a better design of these devices. The dynamic magnetic response of nanoparticles has been extensively analyzed by ferromagnetic resonance (FMR) measurements and the effect of superparamagnetic transition has been highlighted for small nanoparticles [Antoniak 2015]. However, in this kind of experiments conducted at a given frequency when sweeping the magnetic field, the magnetization configuration of the nanoparticles assembly depends on the strength of the static magnetic field making the analysis of experimental FMR spectra complex.

In this work, we performed zero-field microwave permeability measurements from $1 \mathrm{MHz}$ up to $20 \mathrm{GHz}$ to characterize the spectral response of $\mathrm{Zn}_{0.4} \mathrm{Fe}_{2.6} \mathrm{O}_{4}$ nanoparticles and evidence the influence of the superparamagnetic state. The selected chemical composition allows combining a high value of the saturation magnetization with a large electrical resistivity [Fujiwara 2008].

\section{SYNTHESIS OF $\mathrm{Zn}_{0.4} \mathrm{Fe}_{2.6} \mathrm{O}_{4}$ NANOPARTICLES}

A microwave assisted thermal decomposition in organic solvents has been chosen [Liang 2017] because it allows a good control of the nanoparticle size, the size distribution, and the morphology through the tuning of the decomposition temperature, the ratio between precursors and surfactant, the choice of solvent, precursors and surfactant, the heating rate, and the reaction time [Song 2004, $\mathrm{Lu}$ 2007]. In particular, it is really convenient for mixed cubic ferrites $\mathrm{M}_{\mathrm{x}} \mathrm{Fe}_{3-\mathrm{x}} \mathrm{O}_{4}$ synthesis in comparison with other synthetic routes [Pessoa 2019]. However, one parameter remains not well controlled and is clearly affected by the synthesis route: the location of the $\mathrm{M}$ cations in the spinel sites. Indeed, it governs the magnetic properties of the whole structure, making those systems really hard to analyze [Nakagomi 2020].

Iron acetylacetonate (Fe(acac) $)_{3}, 97 \%$ purity), zinc acetylacetonate 
hydrate (Zn(acac) 2 ), oleic acid (OA, 90\% purity) and ethyl acetate were purchased (Sigma-Aldrich), as well as ethylene glycol (EG), absolute ethanol (VWR), 1-octadecene (ODE, Merck Millipore) and oleylamine (OAm, 80-90\% purity, Acros Organics). The following protocol was applied: $1 \mathrm{mmol} \mathrm{Fe}(\text { acac) })_{3}$ and $\left.0.15 \mathrm{mmol} \mathrm{Zn(acac}\right)_{2}$ were added to a homogeneous mixture of $8 \mathrm{~mL} \mathrm{OAm,} 2 \mathrm{~mL} \mathrm{OA}$ and $6 \mathrm{~mL}$ ODE. EG was introduced only to make the nanoparticles grow. The obtained solution was ultrasound-sonicated during 1 hour before introduction in the microwave reactor Monowave 300 (Anton-Paar). The mixture was at first heated up to $473 \mathrm{~K}$ in $5 \mathrm{~min}$ and maintained at this temperature for $10 \mathrm{~min}$. Then, the temperature was increased to $543 \mathrm{~K}$ in $5 \mathrm{~min}$ and maintained for $15 \mathrm{~min}$. The reactor was finally cooled down to $333 \mathrm{~K}$. Finally, the nanoparticles were recovered and washed six times by centrifugation processes with addition of a mixture of ethanol and ethyl acetate. Once all of the organics were removed, the nanoparticles were dried naturally under air. Two categories of nanoparticles were finally studied: small particles (S)P (synthesized without addition of EG in the reactor) and larger ones (L)P (with EG).

\section{EXPERIMENTAL TECHNIQUES}

The nanoparticle morphology and size were observed by transmission electron microscopy (TEM) using a JEOL JEM-2100F TEM with a GATAN UltraScan $4 \mathrm{k}$ camera. The diameter values were obtained from image processing with ImageJ. Sizes were averaged with 5 to 7 samples originating from different synthesis batches and were presented in histograms, applying the Sturges criterion [Aragon 2015]. The average was then calculated from 1000 to 2500 particles by fitting the histograms with a log normal distribution. The phase purity was probed by X-ray diffraction (XRD) with a Malvern PANalytical X'Pert PRO diffractometer using $\mathrm{CuK}_{\alpha}$ line radiation in $\theta / 2 \theta$ configuration and the size of the diffracting domains was calculated with the Scherrer formula.

The magnetic properties were analyzed using a vibrating sample magnetometer (VSM) (Microsense, model EZ7) at room temperature and also with a VSM-SQUID magnetometer (Quantum Design, MPMS) for in-temperature measurements.

Effective magnetic permeability $\mu_{\text {eff }}$ and dielectric permittivity $\varepsilon_{\text {eff }}$ of a cell (APC7 standard) were determined by means of an APC7 coaxial line (Fig. 1.A-C). From the measured transmission and reflection coefficients of a cell containing the nanoparticles, the $\mu_{\text {eff }}$ and $\varepsilon_{\text {eff }}$ spectra were retrieved using the Nicolson-Ross algorithm [Le Gallou 2008]. Two vectorial network analyzers were used: a PNA N5232A from $1 \mathrm{MHz}$ to $6 \mathrm{GHz}$ and a PNA N5230A from $10 \mathrm{MHz}$ to $20 \mathrm{GHz}$ (both from Agilent Technologies) to cover a wide range of frequencies. The APC7 non-magnetic brass cell consists of two lateral plexiglass slices (Fig. 1.D-E) spaced $3 \mathrm{~mm}$ apart with the following properties: a relative permittivity of 2.67 and a thickness of $2.0 \mathrm{~mm}$. The powder is introduced between the two plexiglas slices by keeping one of them as the bottom of the box. The cell is then closed with the second plexiglass slice (Fig. 1.D-E).
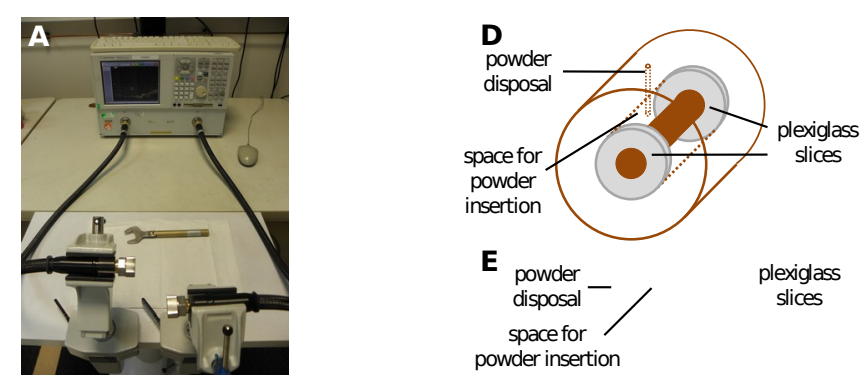

Fig. 1. Set-up for permeability and permittivity measurements (A, B, C), APC7 cell 3D view (D) and cross-sectional view (E).

Measurements of the permeability spectra were also conducted within the [243 K, $343 \mathrm{~K}$ ] range by means of an HP 8720C network analyzer operating from $50 \mathrm{MHz}$ up to $14 \mathrm{GHz}$. The sample is placed in a cell (Fig. 1.D-E) and the whole is then loaded in a furnace to control the temperature. The temperature is then monitored with a thermocouple sensor directly inserted inside the cell. Low temperatures are obtained by a water-cooling circuit.

Based on the volume fraction $f_{V}$ of nanoparticles in the cell, the use of a mixing law is required to determine the intrinsic dynamic permeability from the effective one, considering that the cell contains a mixture of nanoparticles with air. In our case, the retained mixing law is a proportional one with the following formula:

$$
\mu_{\text {tom }}=1+\frac{3}{2 \times f_{\mathrm{v}}}\left(\mu_{\text {eft }}\right.
$$

where $\mu_{\text {int }}$ is the intrinsic permeability. The calculation only regards the transverse dynamic susceptibility noted $\chi_{\perp}$, assuming that the parallel susceptibility is negligible. Thus, the relative permeability $\mu_{r}$ is defined as $\mu_{r}=1+\chi_{\perp}$. The $3 / 2$ prefactor comes from the randomly distributed anisotropy axes within the nanoparticles. It should be remarked that the mixing law does not consider the static and dynamic dipolar coupling between the particles (isolated particles). Taking into account these coupling effects could be achieved through an approach assuming effective demagnetizing factors [Ramprasad 2004].

Considering the low level of measured effective imaginary permeability particularly in the case of the superparamagnetic particles, the uncertainty of measurement, about \pm 0.05 for the cell with Plexiglas sides, has to be taken into account [Bardy 1997].

\section{RESULTS AND DISCUSSION}

\section{A. Nanoparticle size and morphology}

Fig. 2. shows nanoparticles synthesized by thermal decomposition for two ranges of size, namely, SP with a mean diameter of $6.2 \pm 1$ nm exhibiting spherical shapes (Fig. 2.A) and LP with a $22.4 \pm 11$ $\mathrm{nm}$ diameter with "raspberry" morphologies (Fig. 2.B). LP are assimilated to a binding between crystallites and the continuity of the crystal planes in one raspberry structure is noticed. This framework has already been observed in the case of iron oxide [Gerber 2015]. 

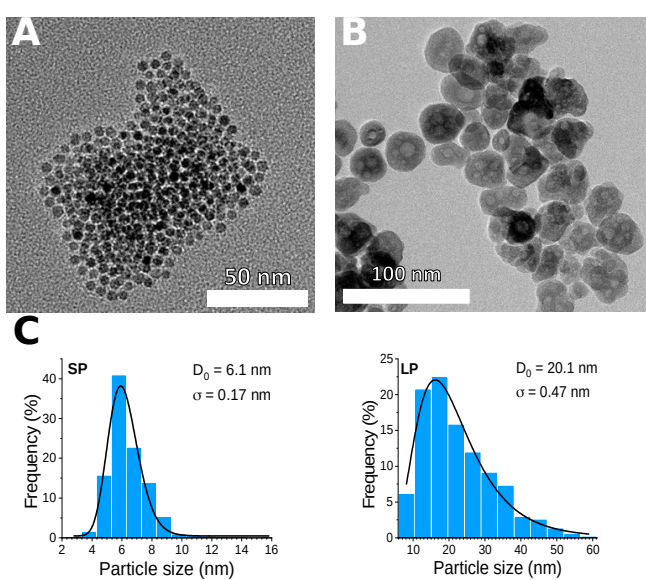

Fig. 2. $\mathrm{Zn}_{0.4} \mathrm{Fe}_{2.6} \mathrm{O}_{4}$ (A) SP and (B) LP obtained by thermal decomposition with sizes of $6.2 \pm 1 \mathrm{~nm}$ and $22.4 \pm 11 \mathrm{~nm}$ respectively. (C) Histogram distributions of the particle size fitted with a lognormal function in blue lines.

The average crystallite size for SP (resp. LP) computed from XRD patterns : $4.5 \mathrm{~nm}$ (resp.19.1 nm) is reduced compared to the TEM one : $6.2 \pm 1 \mathrm{~nm}$ (resp. $22.4 \pm 11 \mathrm{~nm}$ ), as found for $\mathrm{Fe}_{3} \mathrm{O}_{4}$ nanoparticles [Lima 2006].

\section{B. Static magnetic characterization}

In the case of SP the magnetization curve may exhibit, if anisotropy is low enough, a superparamagnetic behavior at room temperature, characterized by a non-saturating signal under high magnetic field. Thus, in the case of a particle assembly, the magnetization direction of each nanoparticle fluctuates over time and the final state is disordered with a zero global magnetization for a measured time above the reversal time of the spins [Dormann 1981]. In this case, a simple model based on the Langevin function has been used to fit the magnetization curve, as follows:

where $\langle M>$ is the average theoretical magnetization, $L$ the Langevin function, $M_{S}$ the saturation magnetization, $\mu_{0}$ the permeability of free space, $H$ the applied magnetic field, $V$ the particle volume, $k$ the Boltzmann constant, and $T$ the temperature [Dormann 1981]. This equation allows recovering the intrinsic saturation magnetization and an apparent size. This procedure is well exemplified on SP, for which Fig. 3.A shows the measured and fitted magnetization curve, at room temperature. The fit provides a $5.4 \mathrm{~nm}$ diameter which could be interpreted as a magnetically ordered volume which is close to the crystallite size (XRD) and smaller than the TEM size.
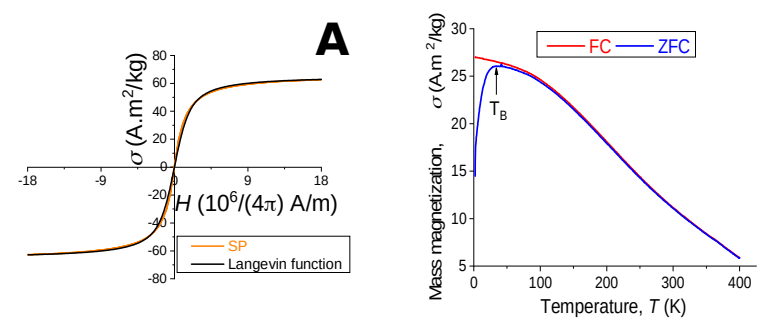

B

Fig. 3. Measured and fitted magnetization curves of SP. Mass magnetization $\sigma$ versus the applied field $H$ (A), ZFC/FC measurement for SP (B).

The superparamagnetic state for $\mathrm{Zn}_{0.4} \mathrm{Fe}_{2.6} \mathrm{O}_{4}$ SP is clearly exhibited by the zero-field cooling/field cooling (ZFC/FC) curve measured with a VSM-SQUID magnetometer as displayed in Fig. 3.B. At first, the nanoparticles are cooled from room temperature to $2 \mathrm{~K}$ at zero field. They are then subjected to a moderate applied field of $500 \times$ $10^{3} /(4 \pi) \mathrm{A} / \mathrm{m}$ and the magnetization is measured during the increase of temperature up to $300 \mathrm{~K}$ (ZFC curve). The same process is done by decreasing the temperature to $2 \mathrm{~K}$ (FC curve) under the same field. It is observed that SP change from the blocked state to the superparamagnetic one at approximatively $33 \mathrm{~K}$, determined from the maximum of the ZFC curve. The FC curve is not constant below $T_{\mathrm{B}}$, which means that dipolar interactions can be neglected in this case [Demortière 2016]. An estimate of the effective anisotropy constant $K$ of nanoparticles can be determined from the ZFC curve. In an uniaxial approximation, the effective anisotropy constant reads [Batlle 1993]:

where $\tau_{m}$ is the measuring time ( $\tau_{m}=2 \mathrm{~s}$ in our case) and $\tau_{0} \sim 1 \times 10^{-9} \mathrm{~s}$ (case of $\mathrm{MnFe}_{2} \mathrm{O}_{4}$ nanoparticles) [Rondinone 2001]. This leads to $K \sim 1.2 \times 10^{5} \mathrm{~J} / \mathrm{m}^{3}$ at $T_{B}=33 \mathrm{~K}$ for nanoparticles with a $5.4 \mathrm{~nm}$ diameter (Fig. 3.A). Moreover, the intrinsic saturation magnetization (65.6 A.m $/ \mathrm{kg}$ ) obtained from (2) is consistent with the one determined at $2 \mathrm{~K}$ using a VSM-SQUID (64.9 A.m²/kg).

\section{Dynamic magnetic characterization}

\section{At room temperature}

Fig. 4 exhibits the permeability spectra for SP and LP. For superparamagnetic SP, the imaginary part of the permeability spectrum reveals a broad resonance spreading between $0.5 \mathrm{GHz}$ and $3.5 \mathrm{GHz}$ (full width at half maximum, FWHM) with a low amplitude. For LP, the resonance amplitude is multiplied by a factor fourteen and the resonance band width extends from $0.5 \mathrm{GHz}$ up to 5 $\mathrm{GHz}$ (FWHM). The level of permeability and the ferromagnetic resonance frequency are drastically modified by the nanoparticles size, hence the superparamagnetic state. This phenomenon could not have been reported by Liang [2017] because these permeability measurements were performed only between 2 and $18 \mathrm{GHz}$. However, the size reduction does not result in a shift of the ferromagnetic resonance towards higher frequencies as suggested by Song [2013].

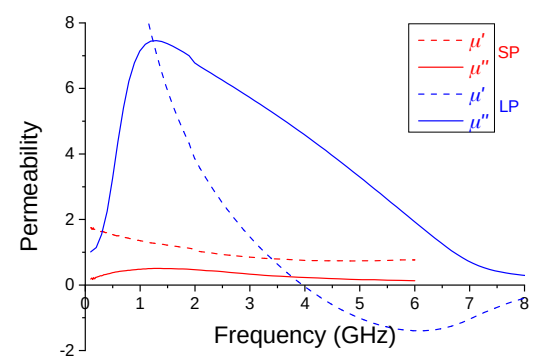

Fig. 4. Permeability spectra for SP and LP. Dashed lines represent the real part of the permeability $\mu^{\prime}$, and solid lines the imaginary part $\mu$ '”.

\section{At various temperatures}

To analyze the dependence of the dynamic permeability through the change of the energy balance between the magnetic anisotropy energy and the thermal energy, permeability measurements have 
been carried out within the temperature range $243 \mathrm{~K}$ to $343 \mathrm{~K}$.

The two sizes of nanoparticles have been considered. Fig. 5 displays the frequency dependence of the imaginary part of the permeability for LP. Two measurements at $293 \mathrm{~K}$ are reported, to compare the results coming from the previous setup (measurements at room temperature) and those arising from the novel one dedicated to the temperature analysis. Keeping in mind the measurement uncertainty, the data show no clear effect of temperature.

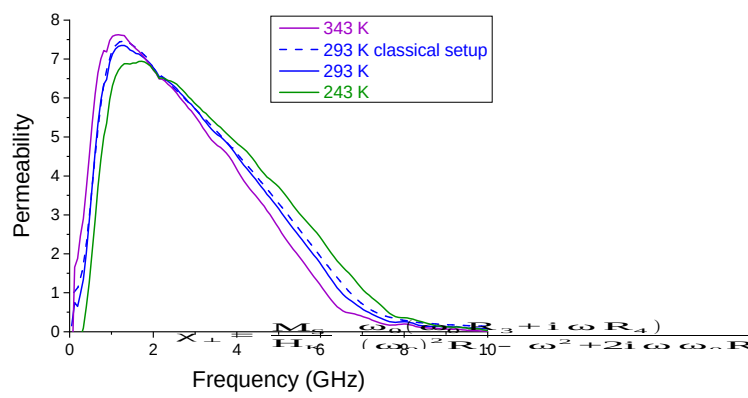

Fig. 5. Evolution of the imaginary part of the permeability spectrum with temperature for $\mathrm{Zn}_{0.4} \mathrm{Fe}_{2.6} \mathrm{O}_{4} \mathrm{LP}$.

For SP (Fig. 6), the imaginary part of the permeability spectrum is significantly modified when the temperature rises from $243 \mathrm{~K}$ to 343 $\mathrm{K}$. The resonance frequency is shifted toward the high frequencies for decreasing temperature. The resonance line intensity defined as the area under the $\mu$ ' ' $(f)$ curve increases significantly for decreasing temperature, which is not observed for LP particles. These results are consistent with those coming from FMR measurements conducted on magnetite [Griscom 1981, Guskos 2005] and maghemite nanoparticles [Gazeau 1998]. Moreover, the width of the resonance peak (FWHM) seems to decrease for decreasing temperature which is in contrast with most of the FMR results. This point would deserve to be deepened through additional temperature dependent permeability measurements. The measurement quality is nevertheless affected by the temperature measurement set-up, especially for the lowest permeability amplitudes which are noisy, that is why the temperature curves displayed in Fig. 6 correspond to smoothed curves treated with the Savitzky-Golay method.

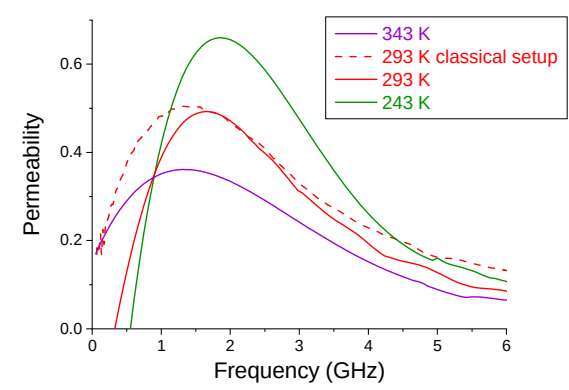

Fig. 6. Evolution of the imaginary part of the permeability spectrum with temperature for $\mathrm{Zn}_{0.4} \mathrm{Fe}_{2.6} \mathrm{O}_{4} \mathrm{SP}$.

\section{Interpretation through a dynamic susceptibility model}

From the theoretical viewpoint, the high-frequency dynamics of superparamagnetic nanoparticles has been extensively studied. The general theoretical approach combines the Landau-Lifshitz-Gilbert equation for the motion of magnetic moment and the Fokker-Planck continuity equation for the orientational distribution function of the magnetic moment [Raikher 2007, Poperechny 2016]. This model has been developed in the context of the FMR where the magnetic moment is subjected to a static polarizing magnetic field. In our experiments, no static magnetic field is applied and the quantity of interest is the zero-field transverse dynamic susceptibility noted $\chi_{\perp}$. As a first approach, the experimental data were interpreted using an approximate analytical model proposed by Raikher [1974]. The main hypotheses are the following. (i) noninteracting randomly distributed nanoparticles are considered. (ii) each particle is assumed spherical, in a single-domain state and possesses an uniaxial magnetic anisotropy. By introducing the ratio between the magnetic anisotropy energy $K V$ and the thermal energy $k T, \sigma=K V / k T$, the zero-field transverse dynamic susceptibility is expressed by:

where $\omega_{0}=\mu_{0} \gamma H_{K}$ is the angular resonance frequency, $\gamma$ the gyromagnetic factor, $H_{K}$ the anisotropy field for an uniaxial particle defined as $H_{K}=2 K /\left(\mu_{0} M_{S}\right), R_{1}, R_{2}, R_{3}, R_{4}$ coefficients from [Raikher, 1974] and depending on $\sigma$, and $\alpha$ the Gilbert damping parameter.
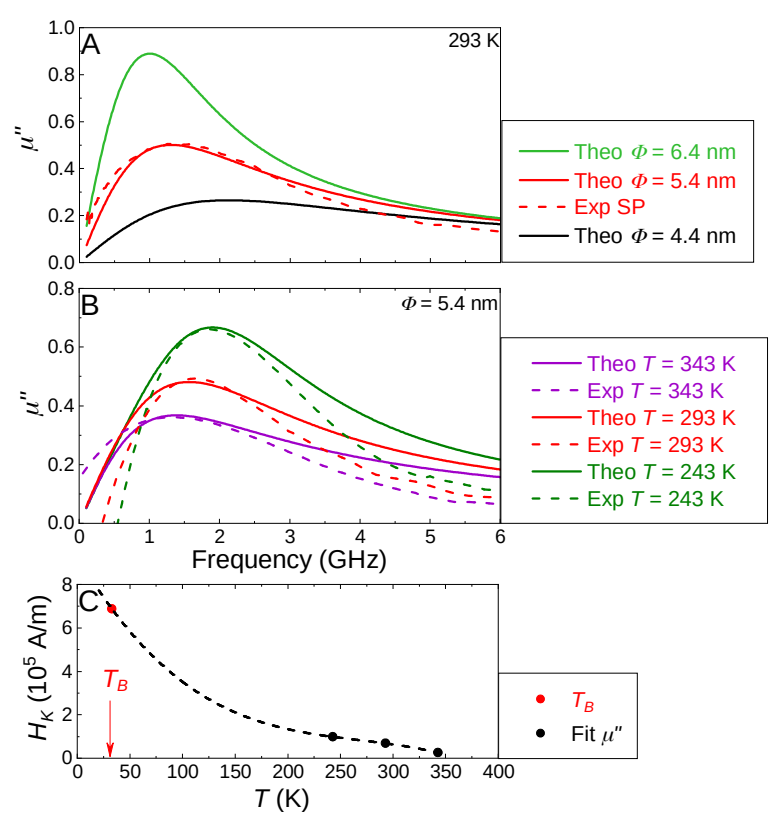

Fig. 7. Computed permeability spectra (imaginary part, $\mu$ '”) and comparison with experiment. (A) Nanoparticle size-dependence of $\mu$ ', at room temperature. The magnetic parameters are: $K=10^{4} \mathrm{~J} / \mathrm{m}^{3}, M_{\mathrm{s}}=3.4410^{5} \mathrm{~A} / \mathrm{m}$, $\gamma=1.76 \times 10^{11} \mathrm{~s}^{-1} \mathrm{~T}^{-1}, \alpha=0.13$. (B) Temperature dependence of $\mu$ "' for $\phi=5.4 \mathrm{~nm}$. The temperature dependence of the effective anisotropy field used for fitting the experimental curves is displayed in (C) (black circles) with the $K$ value at $T_{B}$ (red circle). The dashed line is a guide for eyes.

Fig. 7.A shows the computed nanoparticle size dependence of the permeability spectra (imaginary part) at room temperature. A transition between a relaxant character and a resonant one is revealed for increasing the nanoparticle size. The model reproduces adequately the experimental curve for the nanoparticle diameter $\phi=5.4 \mathrm{~nm}(\sigma \approx 0.2)$ corresponding to the one calculated with the Langevin function (Fig. 3.A) and $K=10^{4} \mathrm{~J} / \mathrm{m}^{3}$. This last value is one order of magnitude lower than the one obtained at $T_{B}$.

Fig. 7.B compares the experimental and theoretical temperature 
dependences of the $\mu$ "' spectra. The experimental evolutions are satisfactorily reproduced by the analytical model, if a temperature dependence of the effective anisotropy field is introduced (Fig. 7.C). Such a rapidly decreasing anisotropy profile has been reported for FePt nanoparticles [Antoniak 2005]. Consequently, this analytical model appears relevant to capture the main features of the experimentally observed superparamagnetic resonances.

\section{CONCLUSION}

Size effects of $\mathrm{Zn}_{0.4} \mathrm{Fe}_{2.6} \mathrm{O}_{4}$ nanoparticles have been exhibited in the zero-field dynamic permeability spectra. The main effect is a drastic reduction of the permeability level, whereas a shift in the resonance frequency is not clearly observed. A dynamic susceptibility model has been used to successfully reproduce the experimental results for the superparamagnetic particles and, in particular, the trend in the temperature dependence of the permeability spectra.

\section{ACKNOWLEDGMENT}

The authors express their appreciation for the support received from Jean-Hugues Le Gallou and Karim Ait Otmane from CEA Le Ripault for the dynamic magnetic measurements, and Silviu Colis from IPCMS for the VSM-SQUID analysis.

\section{REFERENCES}

Aragón F H, Coaquira J A H, Villegas-Lelovsky L, da Silva S W, Cesar D F, Nagamine L C C M, Cohen R, Menendez-Proupin E, Morais P C (2015), "Evolution of the doping regimes in the Al-doped $\mathrm{SnO}_{2}$ nanoparticles prepared by a polymer precursor method,” J. Phys. Condens. Matter, vol. 27, no. 9, pp. 1-7, doi: 10.1088/0953-8984/27/9/095301.

Antoniak C, Lindner J, Farle M (2005), "Magnetic anisotropy and its temperature dependence in iron-rich $\mathrm{Fe}_{x} \mathrm{Pt}_{1-x}$ nanoparticles,” Europhys. Lett. EPL, vol. 70, no. 2, pp. 250-256, doi: 10.1209/epl/i2004-10485-9.

Bañobre-López M, Teijeiro A, Rivas J (2013), “Magnetic nanoparticle-based hyperthermia for cancer treatment,” Rep. Pract. Oncol. Radiother., vol. 18, no. 6, pp. 397-400, doi: 10.1016/j.rpor.2013.09.011.

Bardy N, Leblevennec G, Le Potier D, Authesserre J (1997), “Control of Ferrites Microwave Properties at the French Atomic Energy Commission - Manufacturing Process and Characterization,” J. Phys. IV, vol. 07, no. C1, pp. C1-417-C1-418, doi: 10.1051/jp4:19971168.

Batlle X, García del Muro M, Tejada J, Pfeiffer H, Görnert P, Sinn E (1993), "Magnetic study of $M$-type doped barium ferrite nanocrystalline powders,” J. Appl. Phys., vol. 74, no. 5, pp. 3333-3340, doi: 10.1063/1.354558.

Brown W F (1963), "Thermal Fluctuations of a Single-Domain Particle," Physical Review, vol. 130, no. 5, pp. 1677-1686, doi: 10.1103/PhysRev.130.1677.

Demortière A, Panissod P, Pichon B P, Pourroy G, Guillon D, Donnio B, Bégin-Colin S (2011), "Size-dependent properties of magnetic iron oxidenanocrystals," Nanoscale, vol. 3, no. 1, pp. 225-232, doi: 10.1039/C0NR00521E.

Dormann J L (1981), “Le phénomène de superparamagnétisme,” Rev. Phys. Appliquée, vol. 16, no. 6, pp. 275-301, doi: 10.1051/rphysap:01981001606027500.song

Fujiwara A, Tada M, Nakagawa T, Abe M (2008), "Permeability and electric resistivity of spin-sprayed Zn ferrite films for high-frequency device applications,” J. Magn. Magn. Mater., vol. 320, no. 8, pp. L67-L69, doi: 10.1016/j.jmmm.2007.11.026.

Gazeau F, Bacri J C, Gendron F, Perzynski R, Raikher Y L, Stepanov V I, Dubois E (1998), "Magnetic resonance of ferrite nanoparticles: evidence of surface effects," J. Magn. Magn. Mater., vol. 186, no. 1-2, pp. 175-187, doi: 10.1016/S03048853(98)00080-8.

Gerber O, Pichon B P, Ulhaq C, Grenèche J-M, Lefevre C, Florea I, Ersen O, Begin D, Lemonnier S, Barraud E, Begin-Colin S (2015), "Low Oxidation State and Enhanced Magnetic Properties Induced by Raspberry Shaped Nanostructures of Iron Oxide,” J. Phys. Chem. C, vol. 119, no. 43, pp. 24665-24673, doi: 10.1021/acs.jpcc.5b08164.

Griscom D L (1981), "Particle size effects in the FMR spectra of fine-grained precipitates in glass,” IEEE Trans. Magn., vol. 17, no. 6, pp. 2718-2720, doi: 10.1109/TMAG.1981.1061662.

Guskos N, Anagnostakis E A, Likodimos V, Bodziony T, Typek J, Maryniak M, Narkiewicz U, Kucharewicz I, Waplak S (2005), "Ferromagnetic resonance and ac conductivity of a polymer composite of $\mathrm{Fe}_{3} \mathrm{O}_{4}$ and $\mathrm{Fe}_{3} \mathrm{C}$ nanoparticles dispersed in a graphite matrix,” J. Appl. Phys., vol. 97, no. 2, pp. 1-6, doi: 10.1063/1.1836855.
Le Gallou J-H, Ledieu M, Grimal V, Acher O (2008), “Technique under compressive stress for determining the magneto-elastic effects in ferrites,” J. Magn. Magn. Mater., vol. 320, no. 14, pp. e172-e174, doi: 10.1016/j.jmmm.2008.02.041.

Liang Y-J, Fan F, Ma M, Sun J, Chen J, Zhang Y, Gu N (2017), “Size-dependent electromagnetic properties and the related simulations of Fe3O4 nanoparticles made by microwave-assisted thermal decomposition," Colloids Surf. Physicochem. Eng. Asp., vol. 530, pp. 191-199, doi: 10.1016/j.colsurfa.2017.06.059.

Lima E, Brandl A L, Arelaro A D, Goya G F (2006), "Spin disorder and magnetic anisotropy in Fe3O4 nanoparticles,” J. Appl. Phys., vol. 99, no. 8, pp. 1-10, doi: 10.1063/1.2191471.

Lu A-H, Salabas E L, Schüth F (2007), "Magnetic Nanoparticles: Synthesis, Protection, Functionalization, and Application,” Angew. Chem. Int. Ed., vol. 46, no. 8, pp. 1222-1244, doi: 10.1002/anie.200602866.

Nakagomi F, de Souza P E N, Castro T J, Garg V K, Oliveira A C, e Silva F C, Franco Jr. A, Morais P C, da Silva S W (2020), "Cation distribution of $\mathrm{Zn}_{\mathrm{x}} \mathrm{Co}_{1-\mathrm{x}} \mathrm{Fe}_{2} \mathrm{O}_{4}$ nanoparticles: A resonant X-ray diffraction study,” J. Alloys Compd., vol. 842, pp. 1-10, doi: 10.1016/j.jallcom.2020.155751.

Nikiforov V N, Filinova E Y (2009), Magnetic Nanoparticles, Wiley-VCH, pp. 393444.

Noh S-H, Na W, Jang J-T, Lee J-H, Lee E J, Moon S H, Lim Y, Shin J-S, Cheon J (2012), "Nanoscale Magnetism Control via Surface and Exchange Anisotropy for Optimized Ferrimagnetic Hysteresis,” Nano Lett., vol. 12, no. 7, pp. 3716-3721, doi: 10.1021/nl301499u.

Pessoa M S, Moscon P S, Melo R S, Franco A, Morais P C (2019), “A comprehensive study of $\mathrm{Co}_{1-\mathrm{x}} \mathrm{Ni}_{\mathrm{x}} \mathrm{Fe}_{2} \mathrm{O}_{4}$ nanoparticles fabricated via three different synthetic methods," Mater. Res. Express, vol. 6, no. 12, pp. 1-8, doi: 10.1088/2053-1591/ab597c.

Poperechny I S, Raikher Y L (2016), "Ferromagnetic resonance in uniaxial superparamagnetic particles,” Phys. Rev. B, vol. 93, no. 1, pp. 1-9, doi: 10.1103/PhysRevB.93.014441.

Raikher Y L, Shliomis M I (1974), "Theory of dispersion of the magnetic susceptibility of fine ferromagnetic particles,” Sov. Phys.-JETP, vol. 40, no. 3, pp. 526-532.

Raikher Y L, Stepanov V I (2007), "Magnetization dynamics of single-domain particles by superparamagnetic theory,” J. Magn. Magn. Mater., vol. 316, no. 2, pp. 417421, doi: 10.1016/j.jmmm.2007.03.090.

Ramprasad R, Zurcher P, Petras M, Miller M, Renaud P (2004), “Magnetic properties of metallic ferromagnetic nanoparticle composites,” J. Appl. Phys., vol. 96, no. 1, pp. 519-529, doi: 10.1063/1.1759073.

Rondinone A J, Liu C, Zhang Z J (2001), "Determination of Magnetic Anisotropy Distribution and Anisotropy Constant of Manganese Spinel Ferrite Nanoparticles," J. Phys. Chem. B, vol. 105, no. 33, pp. 7967-7971, doi: 10.1021/jp011183u.

Sai R, Kahmei R D R, Shivashankar S A, Yamaguchi M (2019), "High-Q On-Chip C Band Inductor With a Nanocrystalline MnZn-Ferrite Film Core,” IEEE Trans. Magn., vol. 55, no. 7, pp. 1-4, doi: 10.1109/TMAG.2019.2916699.

Sai R, Kulkarni S D, Yamaguchi M, Bhat N, Shivashankar S A (2017), "Integrated XBand Inductor With a Nanoferrite Film Core,” IEEE Magn. Lett., vol. 8, no. 1, pp. 1-4, doi: 10.1109/LMAG.2017.2655495.

Saleh E, Woolliams P, Clarke B, Gregory A, Greedy S, Smartt C, Wildman R, Ashcroft I, Hague R, Dickens P, Tuck C (2017), “3D inkjet-printed UV-curable inks for multi-functional electromagnetic applications,” Addit. Manuf., vol. 13, pp. 143148, doi: 10.1016/j.addma.2016.10.002.

Song N-N, Yang H-T, Liu H-L, Ren X, Ding H-F, Zhang X-Q, Chenget Z-H (2013), "Exceeding natural resonance frequency limit of monodisperse $\mathrm{Fe} 3 \mathrm{O} 4$ nanoparticles via superparamagnetic relaxation,” Sci. Rep., vol. 3, no. 1, pp. 1-5, doi: 10.1038/srep03161.

Song Q, Zhang Z J (2004), "Shape Control and Associated Magnetic Properties of Spinel Cobalt Ferrite Nanocrystals,” J. Am. Chem. Soc., vol. 126, no. 19, pp. 6164 6168, doi: 10.1021/ja049931r.

Xiao Y, Du J (2020), “Superparamagnetic nanoparticles for biomedical applications,” $J$. Mater. Chem. B, vol. 8, no. 3, pp. 354-367, doi: 10.1039/С9TB01955C. 